\title{
Low-field magnetic characterization of ferromagnets using a minor-loop scaling law
}

\author{
Satoru Kobayashi, ${ }^{1, a)}$ Seiki Takahashi, ${ }^{1}$ Toetsu Shishido, ${ }^{2}$ Yasuhiro Kamada, ${ }^{1}$ and \\ Hiroaki Kikuchi ${ }^{1}$ \\ ${ }^{1}$ NDE and Science Research Center, Faculty of Engineering, Iwate University, Morioka 020-8551, Japan \\ ${ }^{2}$ Institute of Materials Research, Tohoku University, Sendai 980-8577, Japan
}

(Received 31 July 2009; accepted 10 December 2009; published online 25 January 2010)

\begin{abstract}
A scaling power law relating hysteresis loss and remanence in minor hysteresis loops is proposed for the low-field magnetic characterization of ferromagnetic materials. We demonstrate that the law holds true for very low to an intermediate level of magnetization, associated with a universal exponent of $\approx 1.35$, irrespective of types of ferromagnets and temperature, unlike the Steinmetz law with limited applicability. The coefficient in the scaling law shows almost the same behavior as coercivity and can be used for the evaluation of the magnetic quality of materials for which the Steinmetz law cannot be applied and/or low measurement field is necessary.
\end{abstract}

(C) 2010 American Institute of Physics. [doi:10.1063/1.3289317]

\section{INTRODUCTION}

Scaling laws for magnetic minor hysteresis loops have been extensively studied for evaluating the magnetic quality of bulk ferromagnetic materials ${ }^{1-3}$ as well as thin ferromagnetic films. ${ }^{4,5}$ In particular, the power-law relation between hysteresis loss $W_{\mathrm{F}}^{*}$ and the maximum magnetization $M_{\mathrm{a}}^{*}$ of minor hysteresis loops is well known as the Steinmetz law, and is given by

$$
W_{\mathrm{F}}^{*}=W_{\mathrm{F}}^{0}\left(M_{\mathrm{a}}^{*} / M_{\mathrm{s}}\right)^{n_{\mathrm{F}}},
$$

where $n_{\mathrm{F}} \approx 1.6$ and $M_{\mathrm{s}}$ is the saturation magnetization. The coefficient $W_{\mathrm{F}}^{0}$ is a sensitive indicator of the material's intrinsic properties, including magnetocrystalline anisotropy and microstructural properties, such as grain size and dislocation density. The Steinmetz law generally holds true in the intermediate $M_{\mathrm{a}}^{*}$ range where irreversible movement of the Bloch wall plays a dominant role in the magnetization; for $\mathrm{Fe}$ and its alloys, $\mu_{0} M_{\mathrm{a}}^{*}$ is in the range of 0.3-1.2 T.

Our recent systematic studies on $\mathrm{Fe}$ and $\mathrm{Ni}$ single crystals $^{6,7}$ and low-carbon steels ${ }^{8}$ showed that the exponent $n_{\mathrm{F}}$ in Eq. (1) is almost independent of the temperature and the level of deformation. $W_{\mathrm{F}}^{0}$ is more sensitive to the defect density than the coercivity of the major hysteresis loop $H_{\mathrm{c}}$ and is related to mechanical and physical properties such as Vickers hardness and ductile-brittle transition temperature. Therefore, the magnetic method using the scaling rule can be used for evaluation of material degradation because of its sensitivity to lattice defects and low measurements fields (typically less than $4 \mathrm{kA} / \mathrm{m}$ ).

Despite the usability of the Steinmetz law for various soft ferromagnetic materials, however, its applicability is limited. For instance, for polycrystals with strong uniaxial anisotropy, the exponent was observed to deviate significantly from the value of $1.6 ; n_{\mathrm{F}}=1.9$ for pure Co. ${ }^{2}$ This might be attributed to the fact that the minor-loop parameters $W_{\mathrm{F}}^{*}$ and $M_{\mathrm{a}}^{*}$ reflect the average of minor loops of all grains

\footnotetext{
${ }^{a)}$ Electronic mail: koba@iwate-u.ac.jp.
}

which are largely different in shape from grain to grain; since for polycrystal anisotropy axes change from grain to grain, the magnetization process for each grain strongly depends on the relative orientation between the anisotropy axis and applied fields. Similar deviations in $n_{\mathrm{F}}$ occur in the presence of sample inhomogeneities, ${ }^{9,10}$ texture, ${ }^{11}$ etc. Further, the magnetization range in which the Steinmetz law is valid varies according to the material's intrinsic properties as well as external conditions such as temperature and stress. This makes the scaling analysis difficult to perform in practice for the integrity assessment of the ferromagnetic materials.

In this study, we have developed a scaling power law relating hysteresis loss and remanence of minor loops for the characterization of bulk ferromagnetic materials, which was originally proposed for characterizing strain-induced ferromagnetic $\alpha$-martensites in an austenitic matrix, ${ }^{12}$ where large magnetic contribution due to the paramagnetic austenitic phase as well as vacuum permeability by applied fields invalidates the Steinmetz law. The proposed scaling law allows the evaluation of the magnetic quality of bulk ferromagnetic materials in cases where the existing scaling laws cannot be used and/or low measurement field is indispensable.

\section{EXPERIMENT}

We prepared toroidal samples of polycrystals of $\mathrm{Fe}-3$ wt $\% \mathrm{Si}, \mathrm{Ni}$, and $\mathrm{Co}$ with a purity of $99.9 \%$ and grain sizes of 180, 33, and $20 \mu \mathrm{m}$, respectively, as well as Co single crystal grown with electron-beam floating zone melting method. Eighty turns of exciting and pickup coils were wound around the samples with external and internal diameters of 9 and $7 \mathrm{~mm}$, respectively, and thickness $5 \mathrm{~mm}$. The samples were placed in a He-gas closed-cycle refrigerator with a high-temperature stage, by which the temperature was varied from 10 to $700 \mathrm{~K}$. A set of symmetrical minor hysteresis loops with various amplitudes of an applied cyclic field $H_{\mathrm{a}}$ up to $10 \mathrm{kA} / \mathrm{m}$ was measured. Before measuring each minor loop, the sample was demagnetized with a decaying alternating magnetic field. The magnetizing frequencies were 

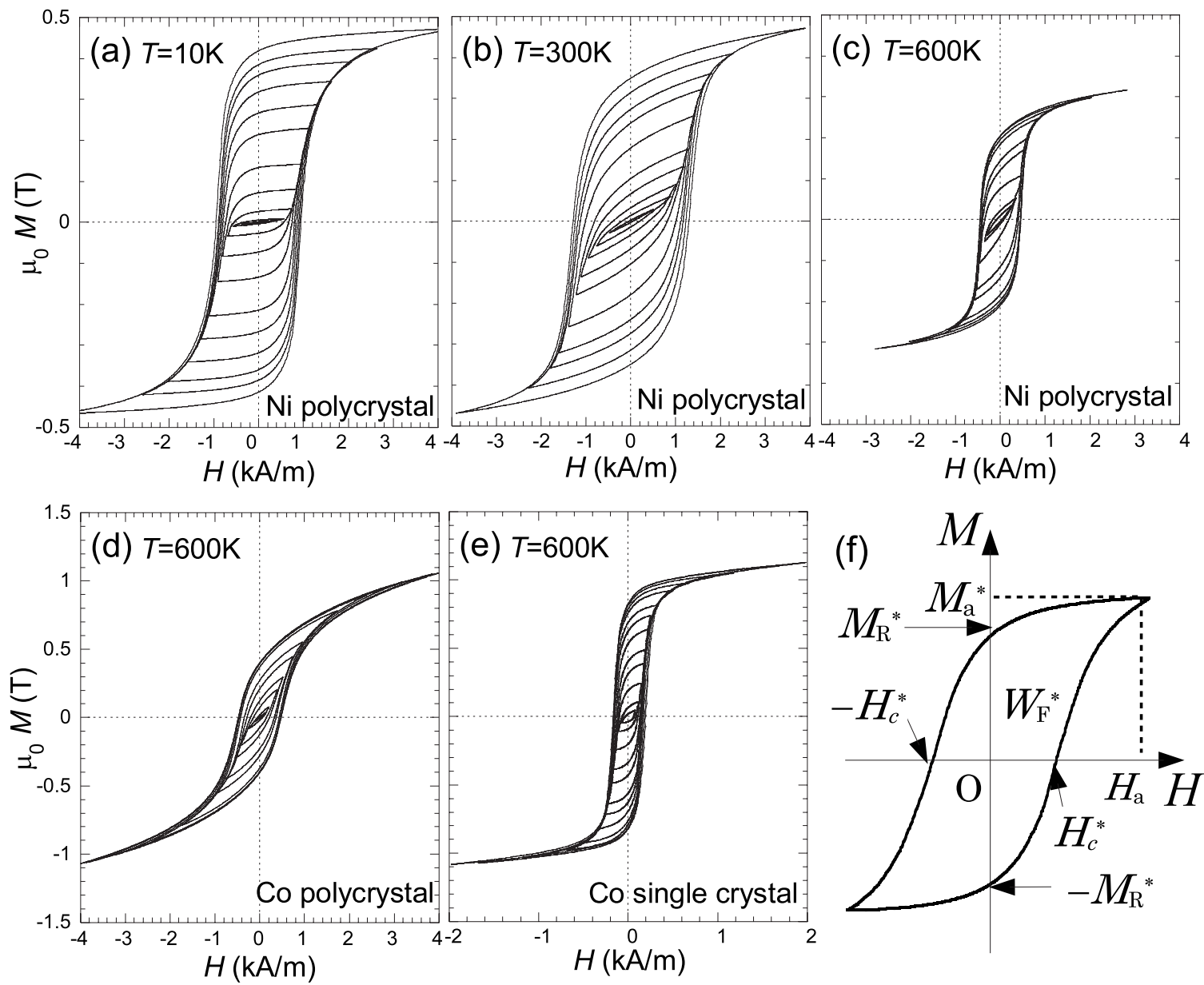

FIG. 1. A set of symmetrical minor hysteresis loops with various field amplitudes, taken at (a) $T=10 \mathrm{~K}$, (b) $300 \mathrm{~K}$, and (c) $600 \mathrm{~K}$ for Ni polycrystals, as well as that taken at $T=600 \mathrm{~K}$ for (d) Co polycrystal and (e) Co single crystal. For clarity, only the representative loops are shown. (f) Parameters of a symmetrical minor hysteresis loop with field amplitude $H_{\mathrm{a}}$, where $W_{\mathrm{F}}^{*}, M_{\mathrm{a}}^{*}, M_{\mathrm{R}}^{*}$, and $H_{\mathrm{c}}^{*}$ represent hysteresis loss, maximum magnetization, remanence, and coercive force of a minor loop, respectively.

$0.01,0.1$, and $0.2 \mathrm{~Hz}$ for the $\mathrm{Fe}-\mathrm{Si}$ alloy, $\mathrm{Ni}$, and Co samples, respectively and the influence of eddy current on the minor loops was negligibly small. Using the set of minor loops, parameters such as $W_{\mathrm{F}}^{*}$ and $M_{\mathrm{a}}^{*}$ [see Fig. 1(f)] were obtained for each minor loop, and the scaling relations were examined in detail.

\section{RESULTS AND DISCUSSION}

As a typical example of a set of symmetrical minor hysteresis loops, the data for Ni polycrystal at several temperatures and for Co polycrystal and single crystal at $T=600 \mathrm{~K}$ are shown in Figs. 1(a)-1(e). For clarity, only the representative loops are shown. Figure 2 show the $H_{\mathrm{a}}$ dependence of coercive force $H_{\mathrm{c}}^{*}$ and hysteresis loss $W_{\mathrm{F}}^{*}$ of minor loops at various temperatures for the $\mathrm{Ni}$ and Co polycrystal samples. One can see that minor loops drastically change in shape with temperature and both $H_{\mathrm{c}}^{*}$ and $W_{\mathrm{F}}^{*}$ are strongly temperature dependent for both the $\mathrm{Ni}$ and Co samples. For the $\mathrm{Ni}$ sample, both $H_{\mathrm{c}}^{*}$ and $W_{\mathrm{F}}^{*}$ at a fixed high value of $H_{\mathrm{a}}$ increase, maximize around $T=200-300 \mathrm{~K}$, and then decrease as the temperature increases toward the Curie temperature of 628 $\mathrm{K}$. In addition, $H_{\mathrm{a}}$ at which the parameters start to steeply increase shifts toward a higher value and then decreases with temperature. These behaviors of minor loops are similar to those for Ni single crystals obtained previously. ${ }^{7}$ For the Co polycrystal, $H_{\mathrm{c}}^{*}$ at a high value of $H_{\mathrm{a}}$ decreases with increasing temperature, whereas $W_{\mathrm{F}}^{*}$ increases, maximizes around $T=500 \mathrm{~K}$, and then decreases. These temperature dependence can be responsible for magnetocrystalline anisotropy of Co, which changes at $T \sim 520 \mathrm{~K}$ from uniaxial to conical anisotropy, and then to basal one at $T \sim 610 \mathrm{~K} .{ }^{13}$ Note that for the Co single crystal pronounced hysteresis loops were only observed around $T=600 \mathrm{~K}$, whereas at the rest of temperatures nearly reversible minor loops due to domain rotation were detected. For the Fe-Si polycrystal, on the other hand, changes in minor loops with temperature are small, reflecting weakly temperature dependent anisotropy and the high Curie temperature (not shown).

Figures 3(a) and 3(b) show the double logarithmic plots of the relation between $W_{\mathrm{F}}^{*}$ and $M_{\mathrm{a}}^{*}$ for $\mathrm{Fe}-\mathrm{Si}$ and Ni polycrystals, respectively, at various temperatures. The $W_{\mathrm{F}}^{*}-M_{\mathrm{a}}^{*}$ curves are linear in the limited intermediate $\mu_{0} M_{\mathrm{a}}^{*}$ range; the slope is weakly temperature dependent for both materials, indicating that these parameters are related by a power law. $\mu_{0} M_{\mathrm{a}}^{*}$ is in the range of $0.2-1.2$ and $0.2-0.4 \mathrm{~T}$ for $\mathrm{Fe}-\mathrm{Si}$ and $\mathrm{Ni}$ polycrystals, respectively; however, the range for $\mathrm{Ni}$ shifts toward lower values at temperatures near $T_{\mathrm{c}}=628 \mathrm{~K}$. Least-squares fits to Eq. (1) yielded $n_{\mathrm{F}}=1.44 \pm 0.01$ and 

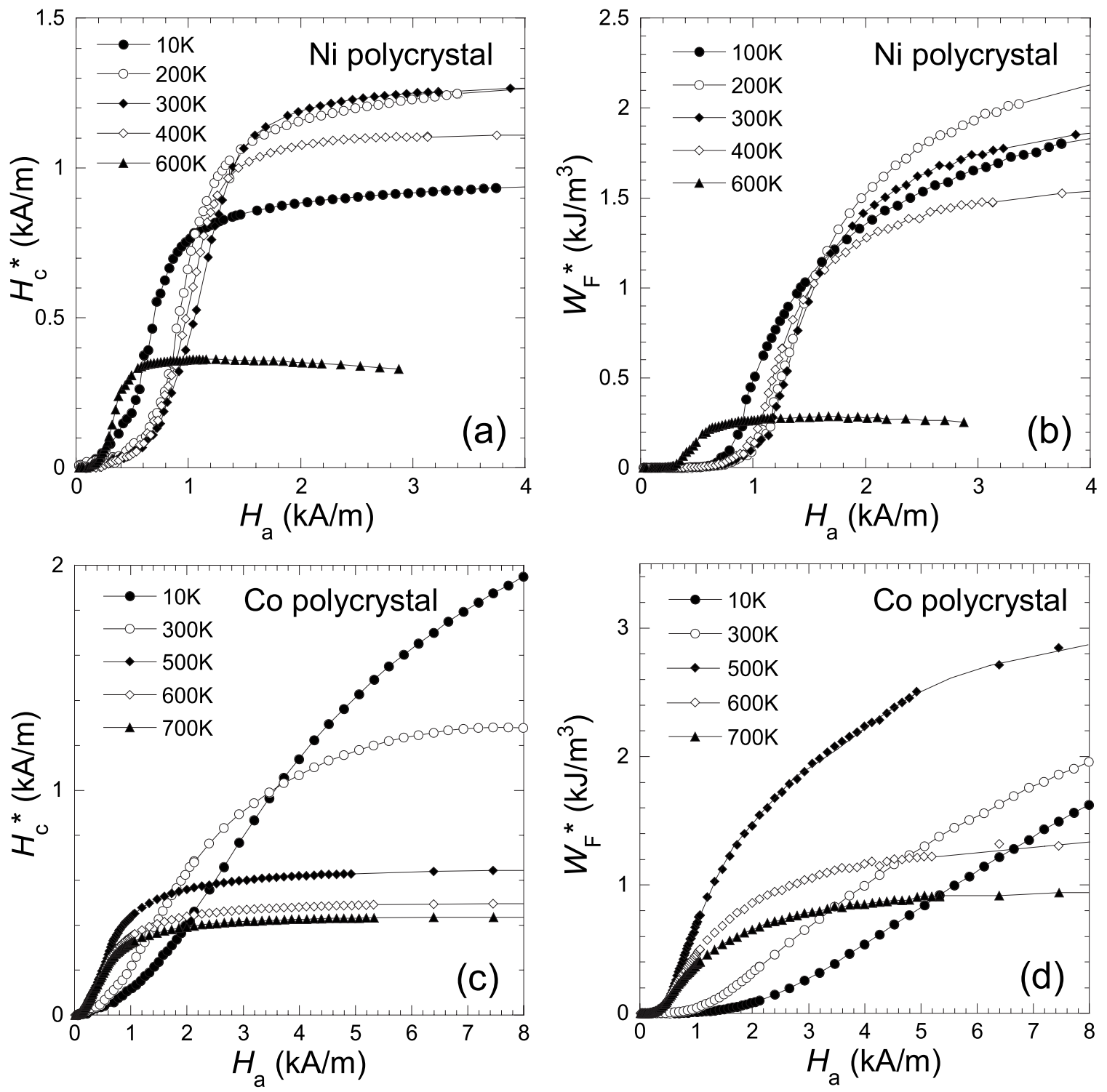

FIG. 2. Minor-loop parameters as a function of $H_{\mathrm{a}}$ at various temperatures; (a) $H_{\mathrm{c}}^{*}$ and (b) $W_{\mathrm{F}}^{*}$ for the Ni polycrystal sample and (c) $H_{\mathrm{c}}^{*}$ and (d) $W_{\mathrm{F}}^{*}$ for the Co polycrystal sample.

$1.5 \pm 0.1$ for $\mathrm{Fe}-\mathrm{Si}$ and $\mathrm{Ni}$, respectively. These values are consistent with the Steinmetz value of 1.6. Even for the Co polycrystal, $W_{\mathrm{F}}^{*}$ and $M_{\mathrm{a}}^{*}$ are related by the power law in a limited range of $\mu_{0} M_{\mathrm{a}}^{*}$, as shown in Fig. 3(c). However, $n_{\mathrm{F}}$ significantly deviates from 1.6 and attains a high value of $2.1 \pm 0.1$ in the wide temperature range of $10-600 \mathrm{~K}$. The value of $n_{\mathrm{F}}$ for the Co polycrystal is dissimilar to $1.43 \mathrm{ob}-$ tained for Co single crystal, as illustrated for $T=600 \mathrm{~K}$ in Fig. 3(c).

Generally, the magnetization process before the saturation level is reached can be divided into three main stages. ${ }^{2}$ In the first stage around the origin, the magnetization process is reversible, while in the second stage, the irreversible movement of the Bloch wall becomes dominant and the magnetization with field shows a steep increase. In the third stage, the magnetization process proceeds gradually due to irreversible domain rotation. It is generally accepted that the Steinmetz law, which is an empirical law, holds true in the second stage and that the irreversible movement of the Bloch wall governs the law. The magnetization range in the second stage strongly depends on the material's intrinsic properties such as crystal symmetry, magnetocrystalline anisotropy, magnetostriction, and microstructures. In the case of hexagonal $\mathrm{Co}$, the crystal is magnetically uniaxial, and its magnetocrystalline anisotropy is one to two orders of magnitude greater than that of bcc $\mathrm{Fe}$ and fcc Ni crystals. ${ }^{2}$ Therefore, for Co polycrystal with anisotropy axes changing from grain to grain, the magnetization process for each grain strongly depends on the relative orientation between the anisotropy and applied fields; for grains having the anisotropy axis nearly parallel to applied fields, the magnetization steeply increases with field due to Bloch wall movement, whereas for grains whose anisotropy axes are not parallel to applied fields the magnetization shows a slow increase due to a considerable contribution of domain rotation. ${ }^{2,13}$ Consequently, when a cyclic field of $H_{\mathrm{a}}$ is applied to the sample, each grain shows a largely different minor hysteresis loop in magnitude and shape, and the macroscopic parameters $M_{\mathrm{a}}^{*}$ and $W_{\mathrm{F}}^{*}$, 

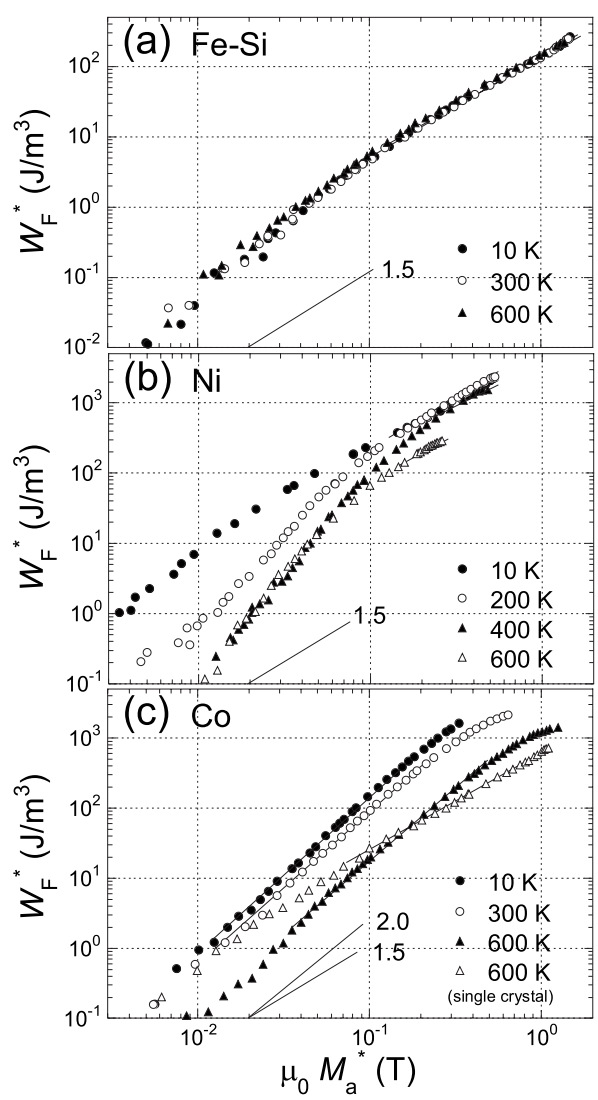

FIG. 3. Relations between $W_{\mathrm{F}}^{*}$ and $M_{\mathrm{a}}^{*}$ for (a) $\mathrm{Fe}-\mathrm{Si}$, (b) $\mathrm{Ni}$, and (c) $\mathrm{Co}$ polycrystals at various temperatures. For comparison, the data for Co single crystal (open triangles) at $T=600 \mathrm{~K}$ are shown in (c). The solid lines through the points represent the least-squares fits.

which reflect the average of minor loops for all grains, would not follow the Steinmetz law. This might be responsible for the large deviation of $n_{\mathrm{F}}$ for the Co polycrystal, as shown in Fig. 3(c).

Now, we consider the scaling relations from the viewpoint of the Rayleigh law. The Rayleigh law ${ }^{14}$ efficiently describes magnetization at low magnetic fields and is expressed as $M=\chi_{\mathrm{a}} H+\frac{1}{2} b H^{2}$, where the initial susceptibility $\chi_{\mathrm{a}}$ and the Rayleigh constant $b$ are parameters related to the reversible and irreversible movement of the Bloch wall, respectively. In some materials such as $\mathrm{Ni}$ and $\mathrm{Co}$, domain rotation also contributes to the first term of the law. The minor-loop parameters $M_{\mathrm{a}}^{*}$ and $W_{\mathrm{F}}^{*}$ that are obtained by applying a cyclic field of amplitude $H_{\mathrm{a}}$ to the materials can be expressed as $\chi_{\mathrm{a}} H_{\mathrm{a}}+b H_{\mathrm{a}}^{2}$ and $\frac{4}{3} \mu_{0} b H_{\mathrm{a}}^{3}$, respectively. Using these parameters, the relation between $W_{\mathrm{F}}^{*}$ and $M_{\mathrm{a}}^{*}$ can be expressed as follows. ${ }^{15}$

$$
W_{\mathrm{F}}^{*}=\mu_{0}\left(-\chi_{\mathrm{a}}+\sqrt{\left(\chi_{\mathrm{a}}\right)^{2}+4 M_{\mathrm{a}}^{*} b}\right)^{3} /\left(6 b^{2}\right)
$$

If the movement of the Bloch wall is reversible, Eq. (2) yields the relation $W_{\mathrm{F}}^{*}=\frac{4}{3} \mu_{0} b\left(M_{\mathrm{a}}^{*} / \chi_{\mathrm{a}}\right)^{3}$, while Eq. (2) can be reduced to $W_{\mathrm{F}}^{*}=\frac{4}{3} \mu_{0} b\left(M_{\mathrm{a}}^{*} / b\right)^{3 / 2}$ if the irreversible movement dominates. Therefore, the relation of Eq. (2) always gives an exponent of 1.5-3 as long as the Rayleigh law holds true. The value of the exponent is generally dependent on the types of ferromagnets, temperature, stress, magnetocrystalline anisotropy, etc. For instance, for Ni polycrystal with the
Rayleigh range of $\mu_{0} M_{\mathrm{a}}^{*}<\sim 0.04 \mathrm{~T}$, the exponent is strongly temperature dependent and close to 3 at higher temperatures because of significant contribution of rotation mechanism, ${ }^{13}$ whereas for $\mathrm{Fe}-\mathrm{Si}$ polycrystal with the Rayleigh range of $\mu_{0} M_{\mathrm{a}}^{*}<\sim 0.05 \mathrm{~T}$, the exponent is about 2 . In the intermediate $M_{\mathrm{a}}^{*}$ range beyond the Rayleigh region, on the other hand, the well-known empirical Steinmetz law holds true. This Steinmetz law can be related to the Rayleigh law with dominant irreversible mechanism, because in this $M_{\mathrm{a}}^{*}$ range the irreversible movement of Bloch wall dominates the magnetization mechanism and the exponent of the Steinmetz law is close to 1.5 predicted by the Rayleigh law. Therefore, it would be always expected that the power-law exponent of the $W_{\mathrm{F}}^{*}-M_{\mathrm{a}}^{*}$ relation shows a transition from $1.5-3$ in the Rayleigh region to about 1.5 in the intermediate $M_{\mathrm{a}}^{*}$ range with increasing maximum magnetization. In contrast, for the Co polycrystal with strong magnetocrystalline anisotropy, the magnetization process is always associated with irreversible domain wall motion and domain rotation and $n_{\mathrm{F}}$ falls in the value between 1.5 and 3 even in the intermediate $M_{\mathrm{a}}^{*}$ range. Therefore, the Steinmetz law has limited applicability with respect to the magnetization range and the types of ferromagnetic materials.

On the other hand, the remanence of minor loops $M_{\mathrm{R}}^{*}$ is given by $M_{\mathrm{R}}^{*}=\frac{1}{2} b H_{\mathrm{a}}^{2}$ and does not include explicitly $\chi_{\mathrm{a}}$. Therefore, the relation between $W_{\mathrm{F}}^{*}$ and $M_{\mathrm{R}}^{*}$ is expressed as

$$
W_{\mathrm{F}}^{*}=4 \mu_{0}\left(2 M_{\mathrm{R}}^{*}\right)^{3 / 2} /\left(3 b^{1 / 2}\right),
$$

and only includes the parameter $b$. This implies that this scaling law will be valid irrespective of the magnetization range as well as the microscopic magnetic state of each grain.

Figures 4(a)-4(c) show the double logarithmic plots of the relation between $W_{\mathrm{F}}^{*}$ and $M_{\mathrm{R}}^{*}$ for $\mathrm{Fe}-\mathrm{Si}, \mathrm{Ni}$, and Co polycrystals, respectively. The curves are linear over a limited range of $M_{\mathrm{R}}^{*}$; however, the linearity persists even for minor loops obtained with very low $H_{\mathrm{a}}$. This indicates that the power law is valid for very low to an intermediate level of magnetization. By assuming the equation

$$
W_{\mathrm{F}}^{*}=W_{\mathrm{m}}^{0}\left(M_{\mathrm{R}}^{*} / M_{\mathrm{R}}\right)^{n_{\mathrm{m}}},
$$

an almost constant value of $n_{\mathrm{m}} \approx 1.35$, which is close to 1.5 derived from the Rayleigh law, was obtained from the leastsquares fits for all the materials. Here, $W_{\mathrm{m}}^{0}$ and $M_{\mathrm{R}}$ are a coefficient and the remanence, respectively. Similar values of $n_{\mathrm{m}}$ were obtained for $\mathrm{Fe}, \mathrm{Ni}$, and Co single crystals. The values and $M_{\mathrm{a}}^{*}$ range for which Eq. (4) holds true are listed in Table I. Note that within the measurement accuracy, the validity of Eq. (4) can be confirmed down to a $\mu_{0} M_{\mathrm{a}}^{*}$ value of the order of $10^{-3} \mathrm{~T}$, except in the case of Ni polycrystal.

Figures 5(a) and 5(b) show $W_{\mathrm{F}}^{0}, W_{\mathrm{m}}^{0}$, and $H_{\mathrm{c}}$ as a function of temperature for $\mathrm{Fe}-\mathrm{Si}$ and $\mathrm{Ni}$ polycrystals, respectively. The variation of $W_{\mathrm{m}}^{0}$ with temperature is almost identical to that of structure-sensitive magnetic properties $W_{\mathrm{F}}^{0}$ and $H_{\mathrm{c}}$ and agrees with the previous observations. ${ }^{13}$ This good relationship can be understood on the basis of the Néel theory, ${ }^{16}$ where the coercivity was related to the parameters of the Rayleigh law using statistical potential function of the Bloch wall pinning. The theory showed that both $\chi_{\mathrm{a}} H_{\mathrm{c}}$ and $b H_{\mathrm{c}} / \chi_{\mathrm{a}}$ 

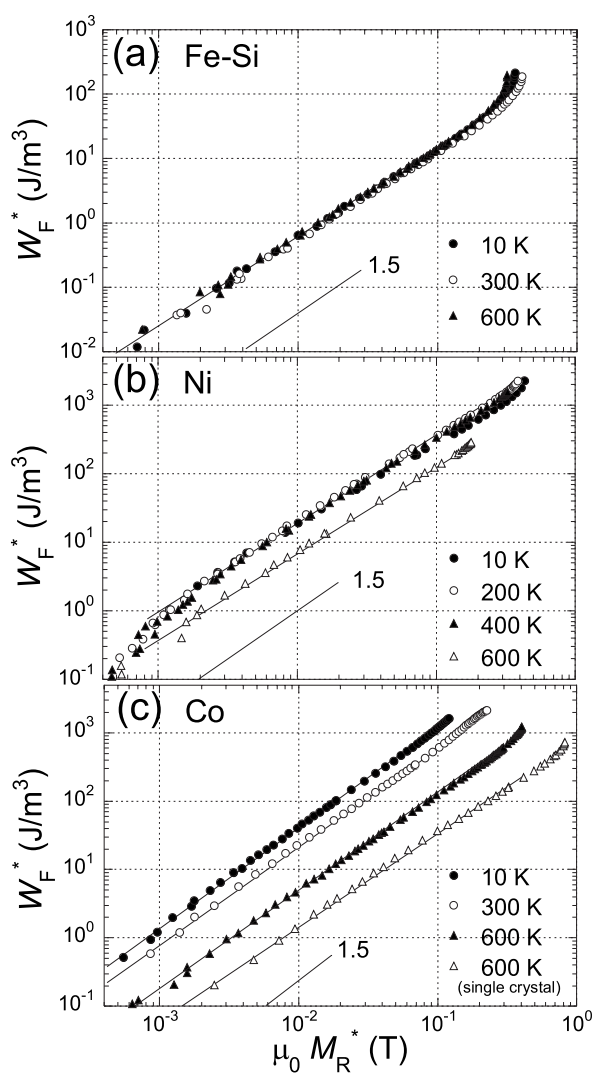

FIG. 4. Relations between $W_{\mathrm{F}}^{*}$ and $M_{\mathrm{R}}^{*}$ for (a) $\mathrm{Fe}-\mathrm{Si}$, (b) $\mathrm{Ni}$, and (c) $\mathrm{Co}$ polycrystals at various temperatures. For comparison, the data for Co single crystal (open triangles) at $T=600 \mathrm{~K}$ are shown in (c). The solid lines through the points represent the least-squares fits.

are constant and $H_{\mathrm{c}} \propto b^{-1 / 2}$. Since $W_{\mathrm{m}}^{0}$ is proportionally related to $b^{-1 / 2}$ [see Eqs. (3) and (4)], a good relationship between $W_{\mathrm{m}}^{0}$ and $H_{\mathrm{c}}$ would always be expected as far as the Rayleigh law is valid as observed experimentally; the same holds true for $W_{\mathrm{F}}^{0}$ when the irreversible motion of the Bloch wall dominates the magnetization process.

In this study, we obtained $n_{\mathrm{m}} \approx 1.35$ for all the investigated ferromagnets. The value is very close to the reported value of 1.4 for ferromagnetic $\alpha$-martensite clusters formed

TABLE I. $n_{\mathrm{F}}$ and $n_{\mathrm{m}}$, obtained from the $W_{\mathrm{F}}^{*}-M_{\mathrm{a}}^{*}$ and $W_{\mathrm{F}}^{*}-M_{\mathrm{R}}^{*}$ relations, respectively, for various types of ferromagnetic materials. Each value is the average of the values obtained over temperature range of 10-600 K. For comparison, the $M_{\mathrm{a}}^{*}$ range at $T=300 \mathrm{~K}$, where Eqs. (1) and (4) hold true is given.

\begin{tabular}{|c|c|c|c|c|}
\hline \multirow[b]{2}{*}{ Materials } & \multicolumn{2}{|c|}{$W_{\mathrm{F}}^{*}-M_{\mathrm{a}}^{*}$ relation } & \multicolumn{2}{|c|}{$W_{\mathrm{F}}^{*}-M_{\mathrm{R}}^{*}$ relation } \\
\hline & $n_{\mathrm{F}}$ & $\begin{array}{c}\mu_{0} M_{\mathrm{a}}^{*} \\
(\mathrm{~T})\end{array}$ & $n_{\mathrm{m}}$ & $\begin{array}{c}\mu_{0} M_{\mathrm{a}}^{*} \\
(\mathrm{~T})\end{array}$ \\
\hline \multicolumn{5}{|c|}{ Polycrystal } \\
\hline $\mathrm{Fe}-\mathrm{Si}$ & $1.44 \pm 0.01$ & $0.2-1.2$ & $1.34 \pm 0.01$ & $<0.35$ \\
\hline $\mathrm{Ni}$ & $1.5 \pm 0.1$ & $0.2-0.4$ & $1.26 \pm 0.03$ & $0.02-0.4$ \\
\hline Co & $2.1 \pm 0.1$ & $0.02-0.2$ & $1.41 \pm 0.03$ & $<0.5$ \\
\hline \multicolumn{5}{|c|}{ Single crystal } \\
\hline $\mathrm{Fe}$ & $1.48 \pm 0.05$ & $0.2-1.0$ & $1.36 \pm 0.02$ & $<0.7$ \\
\hline $\mathrm{Ni}$ & $1.64 \pm 0.03$ & $0.02-0.24$ & $1.39 \pm 0.05$ & $<0.07$ \\
\hline $\mathrm{Co}$ & $1.43 \pm 0.01$ & $0.1-1.1$ & $1.35 \pm 0.04$ & $<0.8$ \\
\hline
\end{tabular}

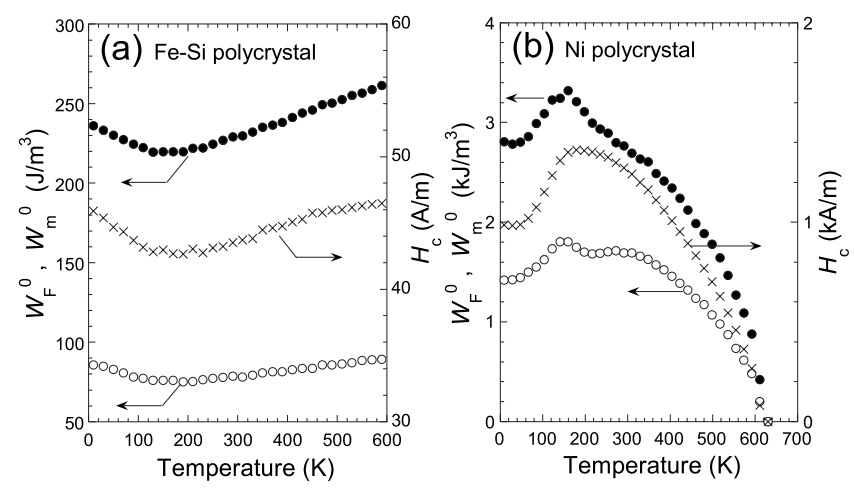

FIG. 5. $W_{\mathrm{F}}^{0}, W_{\mathrm{m}}^{0}$, and $H_{\mathrm{c}}$ as a function of temperature for (a) Fe-Si and (b) $\mathrm{Ni}$ polycrystals, respectively. The solid and open circles and crosses denote $W_{\mathrm{F}}^{0}, W_{\mathrm{m}}^{0}$ and $H_{\mathrm{c}}$, respectively. $H_{\mathrm{c}}$ for the $\mathrm{Fe}-\mathrm{Si}$ and Ni samples was obtained from the major hysteresis loop with $H_{\mathrm{a}}=1.5$ and $8 \mathrm{kA} / \mathrm{m}$, respectively.

in a paramagnetic austenitic matrix. ${ }^{12}$ This means that the exponent $n_{\mathrm{m}}$ is approximately 1.35 universally irrespective of the types of ferromagnetic materials. Although the similar value of 1.5 is derived from the phenomenological Rayleigh law, however, the underlying mechanism that yields the universality and its slightly lower value than 1.5 is not fully understood. Nevertheless, considering that the behavior of minor-loop coefficients as well as the Rayleigh law were well described within the Néel theory, ${ }^{16}$ the statistical properties of the Bloch wall moving in a random pinning field can be connected to the macroscopic scaling power-law between hysteresis loss and remanence of minor loops. Further theoretical investigation is required to clarify this point.

Unlike the Steinmetz law, the scaling law relating hysteresis loss and remanence was found to have two advantages for the magnetic characterization of ferromagnetic materials. One of these advantages is the universality of the exponent being independent of the types of ferromagnets and external conditions. The universality means that the magnetic quality of materials is only reflected in the minor-loop coefficient $W_{\mathrm{m}}^{0}$. Therefore, by obtaining values of $W_{\mathrm{m}}^{0}$ for different materials and/or under different conditions, a difference in magnetic quality due to such as difference in microstructures, defect density, magnetic anisotropy etc. can be accurately evaluated. The other advantage is the wide magnetization range in which the law holds true. As can be seen from Table I, the law covers the magnetization from very low range, for which the Steinmetz law is not valid. This means that the measurement of the structure-sensitive property $W_{\mathrm{m}}^{0}$ requires a far lower applied field than that required for $W_{\mathrm{F}}^{0}$; for instance, in the case of $\mathrm{Fe}-\mathrm{Si}$ polycrystal a maximum field of about $50 \mathrm{~A} / \mathrm{m}$ is enough for obtaining $W_{\mathrm{m}}^{0}$ with good accuracy, in contrast to the field of $\sim 200 \mathrm{~A} / \mathrm{m}$ required for obtaining $W_{\mathrm{F}}^{0}$. This very low measurement field is highly suitable for the in situ integrity assessment of ferromagnetic materials which requires a compact measurement device. The proposed scaling law between hysteresis loss and remanence therefore allows the examination of the magnetic quality of materials for which the existing Steinmetz law cannot be used and/or low measurement field is indispensable. 


\section{SUMMARY}

A scaling relation between hysteresis loss and remanence of symmetrical minor hysteresis loops was examined for polycrystals of $\mathrm{Fe}-\mathrm{Si}, \mathrm{Ni}$, and $\mathrm{Co}$ as well as Co single crystal, varying temperature. We found that the scaling power law with an exponent of $\approx 1.35$ holds true for all the ferromagnets in the wide magnetization range from very low to an intermediate level, while the magnetization range that the Steinmetz law is valid is only limited in the intermediate range and strongly depends both on materials and temperature. The coefficient in the scaling power law shows almost the same behavior as coercivity as well as that of the Steinmetz law. The behavior of the obtained scaling law was explained on the basis of Rayleigh law and Néel theory.

\section{ACKNOWLEDGMENTS}

This research was partly supported by a Grant-in-Aid for Scientific Research (S) (Grant No. 14102034) from the Ministry of Education, Culture, Sports, Science and Technology, Japan. The Co single crystal was prepared under an interuniversity cooperative research program of Advanced Research
Center of Metallic Glasses, Institute for Materials Research, Tohoku University.

${ }^{1}$ C. P. Steinmetz, Proc. IEEE 72, 197 (1984).

${ }^{2}$ R. M. Bozorth, Ferromagnetism (IEEE, New York, 1978) (and references therein).

${ }^{3}$ B. Chakrabarti and M. Acharyya, Rev. Mod. Phys. 71, 847 (1999).

${ }^{4}$ Y. L. He and G. C. Wang, Phys. Rev. Lett. 70, 2336 (1993).

${ }^{5}$ J. S. Suen and J. L. Erskine, Phys. Rev. Lett. 78, 3567 (1997).

${ }^{6}$ S. Takahashi, S. Kobayashi, Y. Kamada, H. Kikuchi, L. Zhang, and K. Ara, Physica B 372, 190 (2006).

${ }^{7}$ S. Kobayashi, T. Fujiwara, S. Takahashi, H. Kikuchi, Y. Kamada, K. Ara, and T. Shishido, Philos. Mag. 89, 651 (2009).

${ }^{8}$ S. Takahashi, S. Kobayashi, H. Kikuchi, and Y. Kamada, J. Appl. Phys. 100, 113908 (2006).

${ }^{9}$ J. D. Ball, Gen. Electr. Rev. 19, 369 (1916).

${ }^{10}$ K. Foster, F. Werner, and R. Del Vecchio, J. Appl. Phys. 53, 8308 (1982).

${ }^{11}$ F. J. G. Landgraf, M. Emura, and M. F. de Campos, J. Magn. Magn. Mater. 320, e531 (2008).

${ }^{12}$ S. Kobayashi, A. Saito, S. Takahashi, Y. Kamada, and H. Kikuchi, Appl. Phys. Lett. 92, 182508 (2008).

${ }^{13} \mathrm{H}$. Träuble, in Magnetism and Metallurgy, edited by A. E. Berkowitz and E. Kneller (Academic, New York, 1969), Chap. 13, p. 621.

${ }^{14}$ L. Rayleigh, Philos. Mag. 23, 225 (1887).

${ }^{15} \mathrm{~F}$. Fiorillo, Measurement and Characterization of Magnetic Materials (Elsevier, Amsterdam, 2004)

${ }^{16}$ L. Néel, Cah. Phys. 12, 1 (1942); 13, 18 (1943). 